\title{
Clinical practice guidelines for insomnia disorder
}

\author{
Lucille Malan, ${ }^{1 *}$ Nokuthula Dlamini ${ }^{2}$ \\ 1 Senior Lecturer, School of Pharmacy, Sefako Makgatho Health Sciences University \\ 2 Junior Lecturer, School of Pharmacy, Sefako Makgatho Health Sciences University \\ *Corresponding author, email: Iucille.malan@smu.ac.za
}

\begin{abstract}
Insomnia disorder is defined as difficulty in falling asleep, maintaining sleep, and early morning awakenings. Common daytime consequences experienced are fatigue, mood instability and impaired concentration. In chronic insomnia these symptoms persist over a period of at least three months. Chronic insomnia can also be a symptom of a variety of disorders. The pathophysiology of insomnia is theorised as a disorder of nocturnal and daytime hyper-arousal as a result of increased somatic, cortical and cognitive activation. The causes of insomnia can be categorized into situational, medical, psychiatric and pharmacologically-induced. To diagnose insomnia, it is required to evaluate the daytime and nocturnal symptoms, as well as psychiatric and medical history. The Diagnostic and Statistical Manual 5 Criteria (DSM-5) also provides guidelines and criteria to be followed when diagnosing insomnia disorder. Goals of treatment for insomnia disorder are to correct the underlying sleep complaint and this, together with insomnia symptoms, their severity and duration, as well as co-morbid disorders will determine the choice of treatment. In the majority of patients, insomnia can be treated without pharmacological therapy and cognitive behavioural therapy is considered first-line therapy for all patients with insomnia. The most common pharmacological insomnia treatments include benzodiazepines and benzodiazepines receptor agonists. To avoid tolerance and dependence, these hypnotics are recommended to be used at the lowest possible dose, intermittently and for the shortest duration possible. A combination of cognitive behavioural therapy and pharmacological treatment options is recommended for chronic insomnia.
\end{abstract}

Keywords: insomnia, benzodiazepines, cognitive behavioural therapy, sleep wake cycle, pharmacologic treatment

\section{Introduction}

According to statistics insomnia is the most common known sleep disorder and $6-10 \%$ of individuals meet the criteria for insomnia disorder. About one-third of adults report having experienced symptoms of insomnia, with $10-15 \%$ recording impaired daytime functioning. Healthy sleep is required for restoring functioning and vitality, maintaining immune function and promoting memory consolidation. However, many individuals are affected with sleep disorders. Untreated sleep disorders can increase the risk of heart disease, memory problems, motor vehicle accidents, and impaired functioning. ${ }^{1-3}$ Furthermore, $40 \%$ of people with insomnia also suffer from psychiatric disorders such as anxiety and depression. ${ }^{4}$ Insomnia is more prevalent in the older population and in a study by the National Institute on Aging, of 9000 patients aged 65 years or older, more than $80 \%$ reported a sleep-related disturbance. ${ }^{4-5}$ Despite being the most prevalent sleep disorder, only $5 \%$ of people suffering from insomnia seek medical help and 10-15\% use non-prescription drugs and alcohol. 5

\section{Classification of Insomnia}

Insomnia disorder is a DSM-5 (The American Diagnostic and Statistical Manual of Mental Disorders, 5th ed.) diagnosis allocated to individuals who experience recurrent poor sleep quantity or quality, leading to anxiety which often causes distress or impairment in important areas of functioning. ${ }^{1,6}$ Categorized as a sleep-wake disorder, insomnia is characterized by difficulty in falling asleep, staying asleep and/or by waking early in the morning and being unable to get back to sleep, which contributes to a sensation of unrefreshing sleep. ${ }^{5-6}$ The DSM-5 Sleep-Wake Disorders Work Group in collaboration with other nosology systems (e.g. International Classification of Sleep Disorders, third edition [ICSD-3]) incorporate changes in diagnoses. The most significant change from the DSM-IV in sleep-wake diagnostic criteria in the insomnia classification is the removal of the distinction between primary and secondary insomnia. Insomnia is recognized as a disorder that needs independent clinical attention, emphasizing the comorbid nature of the disorder and calls for treatment of both insomnia and other medical disorders. The DSM-5 has also included frequency as a diagnostic criterion, and changed the presentation duration for symptoms to be diagnosed as chronic insomnia. ${ }^{1}$ 
Insomnia can further be divided into transient, acute/short term and chronic insomnia.

Transient insomnia is difficulty in sleeping that lasts for a few days or up to one week. It can be caused by anxiety over short term life events leading to acute situational stress, such as a job interview, relationship troubles or conditions such as the flue.?

Acute insomnia, also called adjustment- or stress relatedinsomnia, occurs for a period of less than three months. It is usually associated with more persistent stressful situations caused by stressful life events such as a financial crisis, death or illness of a loved one or change of environment. It commonly resolves without any treatment interventions. ${ }^{8}$

Chronic insomnia is associated with difficulty in sleeping and early morning awakening for more than three nights a week and the symptoms persist for at least three months. It can be associated with a wide variety of disorders. ${ }^{9}$

Symptoms of insomnia disorder can include the following':

- Trouble falling asleep at night

- Lying awake for long periods of time

- Waking several times during the night

- Waking up early and being unable to get back to sleep

- Daytime fatigue and sleepiness

- Inability to concentrate or impaired memory function

- Poor occupational or academic performance, difficulty focusing

- Irritability

- Lack of motivation and energy.

\section{Overview of Normal Sleep}

Sleep is defined as a partial unconcious state whereby stimulation can cause a person to be aroused. This is different from a coma in which a person cannot be aroused by any kind of stimuli. It is a cyclical behaviour that alternates with wakefulness. The cyclical process has two phases, non-rapid eye movement (NREM) and rapid eye movement (REM). ${ }^{10}$ A healthy individual normally has four to six cycles of REME and NREM which last for about 70 to 120 minutes. The two phases are described and distinguished by the patterns they display on the electroencephalogram (EEG). There are 4 stages of NREM and a healthy individual will progress through these stages before the REM period. ${ }^{10}$ Stage 1 and 2 occur during the first 30-45 minutes of sleep and is a state between wakefulness and sleep which can be described as feeling sleepy or drowsy. As we fall deeper into sleep the cycle progress into stages 3 and 4, also called slow wave sleep or delta wave, because of the slow activity of the wave patterns on the EEG. ${ }^{5}$ These waves are characterised by high amplitude and a slow activity. Blood pressure, heart rate and metabolic activity also decrease. After reaching NREM stage 4, occurring about 90 minutes after the commencement of sleep, the EEG wave pattern quickly changes as if backtracking through the stages until alpha waves are reached, indicating the onset of REM sleep. In REM stage the heart rate, blood pressure and respiratory rate increases and the gastrointestinal motility rate decreases. Cerebral blood flow increases and rapid eye movement is seen, but there is still generalized muscle atonia or paralysis which stops us from acting out our dreams. ${ }^{10}$

Table 1: Differences between NREM and REM ${ }^{11}$

\begin{tabular}{ll}
\hline NREM & REM \\
\hline $\begin{array}{l}\text { In adults it constitutes about } \\
\text { 75-80\% of total sleep time }\end{array}$ & Follows after every NREM cycle \\
$\begin{array}{l}\text { Has four stages which cyclically } \\
\text { occur } 4 \text { to } 5 \text { times at night }\end{array}$ & $\begin{array}{l}\text { Characterised by an increased } \\
\text { heart rate, postural muscle atonia } \\
\text { and increased activity on the EEG }\end{array}$ \\
$\begin{array}{l}\text { Non rapid eye movements } \\
\text { which are a sign of quiet } \\
\text { wakefulness occur at stage } 1\end{array}$ & $\begin{array}{l}\text { Most dreams happen during REM } \\
\text { sleep }\end{array}$ \\
$\begin{array}{l}\text { and disappear in the deeper } \\
\text { sleep stages }\end{array}$ & \\
$\begin{array}{l}\text { Stages } 3 \text { and } 4 \text { are referred to } \\
\text { as deep sleep, because arousal } \\
\text { threshold is high (perceived as } \\
\text { high-quality sleep) }\end{array}$ & \\
\hline
\end{tabular}

\section{Circadian Rhythm and Regulation of Sleep}

The sleep cycle indicates a normal circadian rhythm which is regulated by the suprachiasmatic nucleus in the hypothalamus, also called the biological clock. ${ }^{5}$ The suprachiasmatic nucleus induces sleep by inhibiting the reticular activating system through regulation of its preoptic nucleus (sleep inducer) which puts the cortex to sleep..$^{10}$ Orexins, also called the wakeup chemicals are released by the hypothalamus just before waking up and awakens the cortex. ${ }^{10}$ The neurochemistry involved in sleep is complex and difficult to localize to a specific area and neurotransmitter of the brain; however, the NREM phase seems to be controlled by the basal forebrain and the dorsal raphe nucleus which contains most of the serotonergic bodies. ${ }^{5}$ The cholinergic cells in the mesencephalic, medullary, and pontine gigantocellular regions regulate the REM phase. Other neurotransmitters involved in sleep include dopamine, norepinephrine, acetylcholine, histamine and neuropeptides such as substance $P$ and corticotropin-releasing factor. ${ }^{10}$

\section{Etiology and Pathophysiology of Insomnia}

The causes of insomnia can be categorized into situational, medical, psychiatric and pharmacologically induced. Insomnia can be precipitated by underlying medical conditions described in Table $2 .{ }^{5}$ The condition can directly cause insomnia or the symptoms can make it difficult for the patient to sleep (e.g. chronic pain). Approximately $50 \%$ of people suffering from insomnia have a psychiatric disorder, most commonly depressive disorders. ${ }^{12}$

The pathophysiology of insomnia is theorised as a disorder of nocturnal and daytime hyper arousal as a result of increased somatic, cortical and cognitive activation. This can be explained at three levels, cognitive, emotional and physiological. ${ }^{13}$ 


\section{SITUATIONAL}

Personal problems, work related stress, financial crisis, conditions that cause extreme tiredness

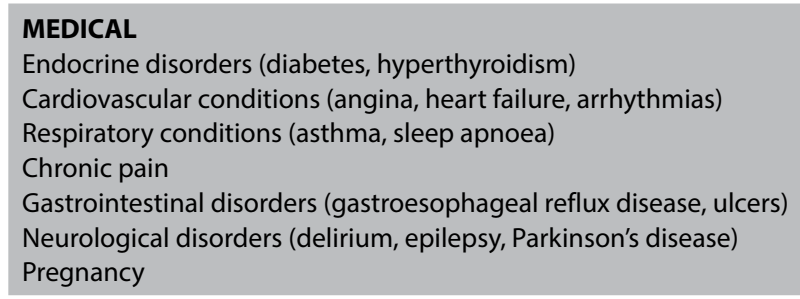

Disorders that affect the mood such as mania and major depressive disorder

Substance abuse

Disorders that cause excessive worrying, nervousness and apprehension, such as generalized anxiety and obsessive compulsive disorder

\section{PHARMACOLOGICALLY INDUCED}

Centrally-acting adrenergic blockers, steroids and stimulants, anticonvulsants, diuretics, selective serotonin reuptake inhibitors

\section{Physiological hyper arousal}

Physiological hyper arousal is associated with increased metabolic activity, temperature, heart rate and increased brain glucose consumption. It is also characterised by high frequency electroencephalographic activity. A study which aimed at examining psychophysiological reactivity to emotional stimuli demonstrated that there was inhibition to facial muscle activity and increased cardiac vagal tone in response to insomnia as compared with good sleepers. ${ }^{9}$

\section{Cognitive hyper arousal}

Cognitive hyper arousal is a result of anxiety and persistent intrusive thoughts which cause hyper vigilance. ${ }^{14}$ The cognitive hyper arousal concept infers that the anxiety associated with lack of sleep leads to increased alertness for sleep related threats (e.g. watching the clock), which indefinitely increases the severity of the condition. ${ }^{9}$

\section{Diagnosis and Evaluation of Insomnia}

Insomnia diagnosis requires evaluation of the daytime and nocturnal symptoms. A full evaluation comprises of a psychiatric and medical history. ${ }^{6}$

\section{History of present illness}

Includes assessing the patient for any insomnia related symptoms. This can be achieved by gathering information like the onset and duration of symptoms. The patient must be asked if there are any events (change of environment, e.g. work, medication use, or a new medical disorder) that coincide with onset of the symptoms. Questions relating to latency of sleep (time it takes to sleep after getting into bed), number and times of awakenings during the night as well as frequency and duration of daytime naps must be asked to evaluate the quality and quantity of sleep. ${ }^{11}$ Other important factors to note are the events which occur just before bedtime, such as physical activity, food and alcohol consumption. Patients must be told to keep a sleep diary where they can detail the events and factors related to insomnia symptoms. ${ }^{11}$

\section{Past medical history}

Involves checking for any medical conditions and use of drugs known to cause insomnia. Disorders that cause insomnia (Table 2) include hyperthyroidism, gastrointestinal conditions, psychiatric disorders, asthma and cardiovascular diseases. ${ }^{11}$

\section{Physical examination}

This is aimed at finding signs related to for example sleep apnoea, to exclude any differential diagnoses. This can include an enlarged uvula, tonsils and tongue, obesity, nasal obstruction and mandibular hypoplasia. ${ }^{11}$

The DSM-5 provides guidelines and criteria to be followed when diagnosing insomnia. Figure 1 shows some of the criteria stipulated in the DSM-IV-TR for diagnosing insomnia, together with the adjusted changes made by the DSM-5. ${ }^{1}$

\section{Managing Insomnia}

Goals of treatment for insomnia disorder are to correct the underlying sleep complaint, being either dissatisfaction with sleep owing to falling asleep, frequent waking during the night or early morning awakening. They also include to improve daytime functioning and sleepiness and avoid side-effects from selected therapies.,5,15 The choice of treatment of insomnia will depend on the specific sleep complaint or insomnia symptoms, their severity and duration, as well as co-morbid disorders. The willingness of the patient to engage in behavioural therapies and the vulnerability of patients to side-effects of medicines will also play a role in managing the disorder. ${ }^{6,16}$

Transient insomnia, occurring as a result of an acute stressor, should resolve with non-pharmacological measures like good sleep hygiene practices and with careful use of sedativehypnotics. In patients presenting with an acute onset insomnia of short duration and with an identifiable precipitant, the Food and Drug Administration (FDA) has approved pharmacological agents for short-term use. However, in chronic insomnia it is recommended that patients receive appropriate treatment for co-existing medical and psychiatric disorders that contribute to the sleeping disorder. ${ }^{5-6}$ Treatable secondary causes of insomnia, for example depression, pain, restless leg syndrome and sleep 


\section{EFFECTS ON QUALITY AND QUANTITY OF SLEEP}

Increased sleep latency

Frequent waking up during the night with difficulty returning to sleep

Early morning awakenings

\section{IMPAIRMENT IN DAYTIME FUNCTIONING}

Poor occupational or academic perfomance Fatigue and sleepiness during the day

Mood changes
Poor concentration

Negative effect on personal relationships and social functioning Behavioural difficulties

\section{IMPAIRMENT IN DAYTIME FUNCTIONING}

Sleep difficulty occurs for at least three nights a week and is present for at least three months

Difficulty in sleeping occurs despite adequate opportunity to sleep

Figure 1: Criteria for Diagnosis of Insomnia Disorder ${ }^{1}$

apnoea, should first be considered, before continuation with insomnia medications. ${ }^{15}$ A two-tiered approach, combining cognitive behavioural therapy (CBT) with pharmacological treatment options are recommended for chronic insomnia.

\section{Non-pharmacological measures}

In the majority of patients insomnia can be treated without pharmacological therapy. ${ }^{5-6,9} \mathrm{CBT}$ is considered first-line therapy for all patients with insomnia, including those with coexisting disorders. ${ }^{15,17-19}$ Studies also indicate that CBT may be more effective than pharmacological therapy for certain measures of insomnia in patients 55 years or older. ${ }^{20-21}$ CBT is most commonly known for sleep-wake behaviour (sleep hygiene) recommendations, stimulus control procedures and cognitive techniques (Table 1). These address dysfunctional behaviours and beliefs about sleep that contribute to the endurance of insomnia. Hence, CBT targets those factors that maintain insomnia over time, such as dysregulation of sleep drive, sleep interfering behaviours and sleep-related anxiety. CBT is usually delivered over the course of 4-8 sessions (30-60 minutes each) that occur weekly or every other week..$^{22}$

The core-components of CBT as described in Table 3 have remained mainly unchanged in the past two decades. The major barrier to the implementation of CBT is the lack of experts available to provide adequate therapy.9.,22 However, recent developments address this limitation by the use of shorter therapies and also include internet-based versions, ${ }^{23}$ abbreviated behavioural treatments ${ }^{24}$ and stepped-care models ranging from self-help to individualised psychotherapy. ${ }^{25}$ These have shown efficacy similar to that of face-to-face CBT. ${ }^{23}$

Disadvantages of CBT are that improvements are typically not seen until 3-4 weeks into treatment and during the first weeks there is often an acute reduction in total sleep time, contributing to increased day-time sleepiness. ${ }^{18}$ Nevertheless, potential advantages include fewer side-effects, and a clear focus on treating the factors that may contribute to chronic insomnia, in an effort to produce long-term positive effects. ${ }^{22}$ Studies have also indicated, that although CBT has a less immediate effect, it is superior to pharmacological treatment when assessed 6-12 months after treatment discontinuation. 19,26

Other approaches with the potential to improve sleep, complementary to psychotherapy, are non-invasive brain stimulation approaches which include thermostimulation and transcranial direct current stimulation. These approaches can induce local activity changes in selected areas of the human cortex and via the cortico-thalamocortical feedback loops; these techniques could modulate arousal and sleep., ${ }^{9,27}$

Education about normal sleep and advice on good sleep hygiene and stimulus control are essential for all patients suffering from insomnia disorder.

\section{Pharmacological treatment options}

Pharmacological treatment for insomnia includes different classes of medications, with differing mechanisms of action, reflecting the multiple neural systems that regulate sleep. The benzodiazepine-receptor agonists are the most widely used agents, and act by strengthening the 'flip-flop sleep switch' between the sleep-wake cycle. ${ }^{6,9,16}$ However, the nonprescription, off-label use of primarily antihistamines, like diphenhydramine, accounts for almost half of the medications routinely used for insomnia. ${ }^{29}$

\section{Benzodiazepine Receptor Agonists}

Benzodiazepines bind to the unique benzodiazepine receptor binding site of the $G A B A_{A}$ receptor complex, resulting in agonistic effects on GABAergic transmission and hyperpolarization of neuronal membranes. The increase in intrinsic activity of the inhibitory neurotransmitter GABA enhances inhibitory outputs to all the major cell groups in the brainstem and hypothalamus that promote arousal. Benzodiazepines enhance the sleeppromoting effects of homeostatic sleep-drive and decrease activity in the arousal systems (e.g. histamine, serotonin acetylcholine and orexin). They therefore also relieve insomnia by reducing sleep latency and increasing total sleep time..$^{5-6,9,16}$ 
Table 3: Components of cognitive behavioural therapy for management of insomnia disorder $5-6,15,28$

\begin{tabular}{ll}
\hline Sleep hygiene & Increase exercise to 3-4 times weekly (not close to bedtime) \\
- Limit intake of alcohol, caffeine and nicotine \\
- Create a comfortable sleep environment; keep bedroom dark, avoid extreme temperatures and loud noises, remove \\
bedroom clocks from sight \\
- Avoid drinking large quantities of liquids in the evening to prevent night-time trips to the bathroom \\
- Eat a light snack before bedtime, not a large meal \\
Stimulus control $\quad$ - Maintain a regular sleep cycle (establish regular times to wake up and go to sleep; including weekends) \\
- Leave the bed when unable to sleep for more than 15-30 minutes, and perform a non-stimulating activity (e.g. read, listen \\
- to soft music) until drowsy \\
- Sleep only as much as necessary to feel refreshed \\
- Sleep-restriction: reduce time in bed to perceived total sleep time (not less than 5-6 hours), avoid daytime or evening \\
naps
\end{tabular}

The benzodiazepine receptor agonists include the traditional benzodiazepines (e.g. oxazepam, flurazepam, quazepam, estazolam, temazepam and triazolam) and the nonbenzodiazepines (e.g. zolpidem, zaleplon and eszopiclone) which act on the same receptor as the benzodiazepines, but without the traditional chemical structure. ${ }^{5-6}$

Benzodiazepines are classified as being long-acting, intermediate-acting, short-acting or ultra-short acting according to their plasma half-lives. Due to benzodiazepines varying predominantly in their half-life, the specific choice of drug is usually based on the insomnia symptom (e.g. difficulty initiating sleep against difficulty maintaining sleep) and the onset of action. ${ }^{6,15}$ Benzodiazepines have a number of potential acute adverse-effects described together with their different half-lives and time of onset in Table $4 .{ }^{30}$

The benzodiazepines are FDA-approved for insomnia, intended for short-term use and patients should be discouraged from using these medicines for extended periods. The FDA also recommends lower doses of benzodiazepines and the nonbenzodiazepines in women and in geriatrics. Benzodiazepines are not approved or recommended for chronic insomnia use, and very long-acting benzodiazepines are not recommended due to risk of daytime side-effects. ${ }^{6,15}$

The non-bezodiazepine, zolpidem (available in SA) and zaleplon or short- or medium-acting benzodiazepines will be first-line therapy for acute insomnia with difficulties in falling asleep. Zolpidem does not appear to have significant effects on next-day psychomotor performance, it is less disruptive of sleep stages, than bezodiazepine hypnotics, as well as associated with less withdrawal, tolerance and rebound insomnia., ${ }^{5,15}$ Oxazepam is the recommended drug of choice by the EML ${ }^{28}$ and is an example of a drug that can be used if patients wake during early hours. Regular reassessment of the benefits and risks is recommended for benzodiazepine receptor agonists and if discontinuation is indicated, gradual, supervised tapering in combination with CBT for insomnia is recommended for chronic users. ${ }^{16,29}$

\section{Miscellaneous Agents (antihistamines, Sedating antidepressants)}

The off-label use of sedating antidepressants and the use of antihistamines are common for insomnia management. Antihistamines, such as diphenhydramine and doxylamine are generally safe and effective for treating mild insomnia. Patients however quickly experience tolerance to the sedative effects and the anticholinergic side-effects such as constipation, especially in the elderly. ${ }^{31-32}$

Antidepressants are alternatives to benzodiazepines, especially for patients who have depression or are at risk of substance abuse. The sedating antidepressants, such as amitriptyline and doxepin are effective for inducing sleep continuity, taking advantage of the antihistaminergic, anticholinergic and serotonergic and adrenergic antagonistic activity of these agents. ${ }^{12,32}$ Doxepin, a tricyclic antidepressant, is FDA-approved for the treatment of insomnia at dosages of 3-6 mg and has shown significant effects on sleep maintenance, but no benefit for sleep-onset latency beyond two days of treatment. ${ }^{31}$ Mirtazepine is another sedating antidepressant that may help patients sleep, but also causes daytime sedation and weight gain. Trazodone (generally at doses of 25-100 mg) can also improve sleep continuity and is popular

Table 4: Pharmacokinetics and side-effects of Benzodiazepine receptor agonists used for Insomnia disorder ${ }^{30}$

\begin{tabular}{llccc}
\hline & Medication & $\begin{array}{c}\text { Half-life } \\
\text { (Hours) }\end{array}$ & $\begin{array}{c}\boldsymbol{t}_{\text {max }} \mathbf{b}^{\mathbf{b}} \\
\text { (Hours) }\end{array}$ & Most common side-effects \\
\hline $\begin{array}{l}\text { Benzodiazepine } \\
\text { Hypnotics }\end{array}$ & $\begin{array}{l}\text { Ultra-short acting: triazolam, } \\
\text { midazolam } \\
\text { Short-acting: oxazepam, }\end{array}$ & $<6$ & $\begin{array}{l}\text { Somnolence (daytime sedation), ataxia, anterograde } \\
\text { amnesia, complex sleep-related behaviours (e.g. } \\
\text { sleepwalking) }\end{array}$ \\
& $\begin{array}{l}\text { temazepam } \\
\text { Intermediate- acting: } \\
\text { alprazolam, lorazepam }\end{array}$ & $12-24$ & 2 & 1.5 \\
$\begin{array}{l}\text { Non-benzodiazepine } \\
\text { receptor agonists }\end{array}$ & $\begin{array}{l}\text { Zolpidem } \\
\text { Zaleplon }\end{array}$ & $2-2.6$ & 1.6 & $\begin{array}{l}\text { Somnolence, dizziness, headache, nausea, abdominal } \\
\text { pain, disturbance in attention }\end{array}$ \\
\hline
\end{tabular}

a Half-life of parent drug

b Time to peak plasma concentration 
for treating insomnia in patients prone to substance abuse and bupropione-induced insomnia, as trazodone does not cause dependence. Other side-effects that might be experienced include morning sedation, orthostatic hypotension (at higher doses) and priapism. Trazodone has also shown efficacy for insomnia co-occurring with major depression. ${ }^{33}$

Few data support the use of these agents, although they have been increasingly used. These drugs are associated with substantial side-effects, such as rebound insomnia after withdrawal, arrhythmia's and liver dysfunction. They are not approved for long-term use and may require medical monitoring; however, the major advantage of these agents is the absence of dependency. ${ }^{9,34}$

\section{Melatonin Receptor Agonist (Ramelteon)}

Ramelteon is a melatonin receptor agonist and FDA- approved for treatment of sleep-onset insomnia. ${ }^{35}$ It is selective for the MT1 and MT2 melatonin receptors and thought to regulate the circadian rhythm and sleep-onset. Ramelteon is associated with reduced subjective sleep latency and improved sleep quality efficiency. ${ }^{36}$ It is proven to effectively treat sleep-onset difficulties in patients with sleep-apnoea and chronic obstructive pulmonary disease. The most common adverse events are somnolence, headache, an unpleasant taste and dizziness; however, ramelteon is generally well-tolerated. ${ }^{35,37-38}$

\section{Orexin Receptor Antagonist (Suvorexant)}

The novel pharmacological agent suvorexant, an orexin receptor antagonist, was approved by the FDA in 2014 for treatment of insomnia disorder. Orexin is a peptide produced by thousands of neurons located in the posterior lateral hypothalamus. These neurons project to the same targets that GABA inhibits and orexin thus leads to diminished activity in sleep-promoting areas, enhancing wakefulness, as well as being involved in the stabilisation of the sleep-wake cycle. Suvorexant acts on this orexin system by supressing the wake-stimulatory network of sleep-wake regulation, leading to decreased time to sleep onset, decreased time awake after sleep onset and increased total sleep time. This is in contrast to the traditional benzodiazepine pharmacological approach, which is to strengthen the sleep drive by augmentation of GABAergic activity. Important risks, such as the risk of dependency and tolerance might thus be avoided. ${ }^{39-42}$ The recommended suvorexant dosage range is 10-20 mg, due to concerns about dose-related, next-day adverse effects, like sedation and cognitive and behavioural changes, such as amnesia and anxiety. ${ }^{43}$

\section{Conclusion}

Increased awareness for effective insomnia disorder treatment is fundamentally important. The goal of treatment for insomnia is to improve sleep and alleviate anxiety or dysfunction caused by the disorder. Management of insomnia in all patients should include identifying the cause of insomnia and possible coexisting disorders. Insomnia can be managed with psychological therapy, which includes CBT, pharmacological therapy or a combination of both. Deciding whether to use pharmacological or behavioural therapy is difficult, as both have their limitations and advantages. Research has shown that CBT was effective in treating the general population of adults, as well as elderly patients with chronic insomnia disorder. Furthermore, CBT is non-invasive and has been shown to likely have fewer harms, such as addiction to pharmacological agents. Medications, however, usually produce rapid improvements and are widely available, but the risk of adverse-effects (like daytime sedation) and risk of dependence may complicate their use. Tolerance and dependence can be avoided by using hypnotics only for the shortest duration possible at the lowest possible dose, intermittently. Patient education about the frequency of drug use and the expected duration of therapy can also help prevent development of dependence.

\section{References}

1. American Psychiatric Association. Diagnostic and Statistical Manual of Mental Disorders. 5th ed. Arlington, VA: American Psychiatric Publishing; 2013.

2. Morin CM, Benca R. Chronic insomnia. The Lancet. 2012;379(9821):1129-41.

3. Walsh JK, Engelhardt CL. The direct economic costs of insomnia in the United States for 1995. Sleep. 1999;22:S386-93.

4. Levenson JC, Kay DB, Buysse DJ. The pathophysiology of insomnia. Chest. 2015;147(4):1179-92.

5. DiPiro JT, Talbert RL, Yee GC, Matzke GR, Wells BG, Posey LM. Pharmacotherapy: A Pathophysiologic Approach, 10th ed. New York, NY: McGraw-Hill;

6. Winkelman JW. Insomnia disorder. New Engl J Med. 2015;373(15):1437-44.

7. Yang CM, Lin SC, Cheng CP. Transient Insomnia Versus Chronic Insomnia: A Comparison Study of Sleep-Related Psychological/Behavioral Characteristics. J Clin Psychol. 2013;69(10):1094-107.

8. Morin CM. Definition of acute insomnia: diagnostic and treatment implications; 2012.

9. Riemann D, Spiegelhalder K, Feige B, Voderholzer U, Berger M, Perlis M, et al. The hyperarousal model of insomnia: a review of the concept and its evidence. Sleep Med Rev. 2010;14(1):19-31.

10. Marieb E, Hoehn K. Human anatomy \& physiology. 9th ed. 2013.

11. Merck \& Co. The Merck manual of diagnosis and therapy. 9th ed. Whitehouse Station: Merck, Rahway, NJ;

12. American Academy of Sleep Medicine. International Classification of Sleep Disorders: Diagnostic and Coding Manual. 3rd ed. Westchester, III: American Academy of Sleep Medicine; 2014.

13. Bonnet MH, Arand DL. Hyperarousal and insomnia: state of the science. Sleep Med Rev. 2010;14(1):9-15.

14. Outhoff K. Insomnia disorder: a hard day's night: review. SA Pharmaceutical Journal. 2016;83(1):14-6.

15. Qaseem A, Kansagara D, Forciea MA, Cooke M, Denberg TD, for the Clinical Guidelines Committee of the American College of Physicians. Management of Chronic Insomnia Disorder in Adults: A Clinical Practice Guideline from the American College of Physicians. Ann Intern Med. 2016;165(2):125-33.

16. Morin $C M$, Edinger JD, Krystal AD, Buysse DJ, Beaulieu-Bonneau S, Ivers $H$. Sequential psychological and pharmacological therapies for comorbid and primary insomnia: study protocol for a randomized controlled trial. Trials. 2016;17(1):118

17. Schutte-Rodin S, Broch L, Buysse D, Dorsey C, Sateia M. Clinical guideline for the evaluation and management of chronic insomnia in adults. J Clin Sleep Med. 2008;4(5):487-504.

18. Edinger JD, Olsen MK, Stechuchak KM, Means MK, Lineberger MD, Kirby A, et al. Cognitive behavioral therapy for patients with primary insomnia or insomnia associated predominantly with mixed psychiatric disorders: a randomized clinical trial. Sleep. 2009;32(4):499-510.

19. Morin CM, Bootzin RR, Buysse DJ, Edinger JD, Espie CA, Lichstein KL. Psychological and behavioral treatment of insomnia: update of the recent evidence (1998-2004). Sleep. 2006;29(11):1398.

20. Morgenthaler $T$, Kramer $M$, Alessi $C$, Friedman $L$, Boehlecke B, Brown $T$, et al. Practice parameters for the psychological and behavioral treatment of 
insomnia: an update. An American Academy of Sleep Medicine report. Sleep. 2006;29(11):1415.

21. Sivertsen B, Omvik S, Pallesen S, Havik OE, Kvale G, Nordhus IH. Cognitive behavioral therapy vs zopiclone for treatment of chronic primary insomnia in older adults: a randomized controlled trial. J Am Med Assoc. 2006;295(24):2851-8.

22. Mitchell MD, Gehrman P, Perlis M, Umscheid CA. Comparative effectiveness of cognitive behavioral therapy for insomnia: a systematic review. BMC Family Practice. 2012;13(1):40.

23. Ritterband LM, Thorndike FP, Gonder-Frederick LA, Magee JC, Bailey ET, Saylor DK, et al. Efficacy of an Internet-based behavioral intervention for adults with insomnia. Arch Gen Psychiatry. 2009;66(7):692-8.

24. Troxel WM, Germain A, Buysse DJ. Clinical management of insomnia with brief behavioral treatment (BBTI). Behav Sleep Med. 2012;10(4):266-79.

25. Espie CA. Stepped care: a health technology solution for delivering cognitive behavioral therapy as a first line insomnia treatment. Sleep. 2009;32(12):1549-58.

26. Morin $C M$, Vallières $A$, Guay B, Ivers $H$, Savard J, Mérette $C$, et al. Cognitive behavioral therapy, singly and combined with medication, for persistent insomnia: a randomized controlled trial. J Am Med Assoc. 2009;301(19):2005-15.

27. Marshall L, Helgadóttir $H$, Mölle $M$, Born J. Boosting slow oscillations during sleep potentiates memory. Nature. 2006;444(7119):610-3.

28. The National Department of Health. Standard Treatment Guidelines and Essential Drug List. Hospital Level Adult GUIDELINES. $2012 \mathrm{ed}$

29. Morin CM, Koetter U, Bastien C, Ware JC, Wooten V. Valerian-hops combination and diphenhydramine for treating insomnia: a randomized placebo-controlled clinical trial. Sleep. 2005;28(11):1465.

30. South African Medicines Formulary. Division of Clinical Pharmacology, Faculty of Health Sciences, University of Cape Town. 12th ed. 2016.

31. Krystal AD, Lankford A, Durrence HH, Ludington E, Jochelson P, Rogowski R, Roth T. Efficacy and safety of doxepin 3 and $6 \mathrm{mg}$ in a 35-day sleep laboratory trial in adults with chronic primary insomnia. Sleep. 2011;34(10):1433.

32. Lippmann S, Mazour I, Shahab H. Insomnia: therapeutic approach. South Med J. 2001;94(9):866.
33. Bertisch SM, Herzig SJ, Winkelman JW, Buettner C. National use of prescription medications for insomnia: NHANES 1999-2010. Sleep. 2014;37(2):343-9.

34. Buscemi N, Vandermeer B, Friesen C, Bialy L, Tubman M, Ospina M, et al. The efficacy and safety of drug treatments for chronic insomnia in adults: a meta-analysis of RCTs. J Gen Intern Med. 2007;22(9):1335.

35. Belsomra, suvorexant [Product Information]. Whitehouse Station, NJ: Merck and Co., Inc.; 2014.

36. Kuriyama $A$, Honda $M$, Hayashino $Y$. Ramelteon for the treatment of insomnia in adults: a systematic review and meta-analysis. Sleep Med. 2014;15(4):385-92.

37. Ferracioli-Oda E, Qawasmi A, Bloch MH. Meta-analysis: melatonin for the treatment of primary sleep disorders. PloS one. 2013;8(5):e63773.

38. Kryger M, Roth $T$, Wang-Weigand $S$, Zhang J. The effects of ramelteon on respiration during sleep in subjects with moderate to severe chronic obstructive pulmonary disease. Sleep Breath. 2009;13(1):79-84.

39. Citrome L. Suvorexant for insomnia: a systematic review of the efficacy and safety profile for this newly approved hypnotic-what is the number needed to treat, number needed to harm and likelihood to be helped or harmed? Int J Clin Pract. 2014;68(12):1429-41.

40. Zeitzer JM, Nishino S, Mignot E. The neurobiology of hypocretins (orexins), narcolepsy and related therapeutic interventions. Trends Pharmacol Sci. 2006;27:368-74.

41. Herring WJ, Connor KM, Ivgy-May N, Snyder E, Liu K, Snavely DB, et al. Suvorexant in patients with insomnia: results from two 3-month randomized controlled clinical trials. Biol Psychiatry. 2016;79(2):136-48.

42. Broese M, Riemann D, Hein L, Nissen C. a-Adrenergic receptor function, arousal and sleep: mechanisms and therapeutic implications. Pharmacopsychiatry. 2012;45(06):209-16.

43. Michelson D, Snyder E, Paradis E, Chengan-Liu M, Snavely DB, Hutzelmann J, et al. Safety and efficacy of suvorexant during 1-year treatment of insomnia with subsequent abrupt treatment discontinuation: a phase 3 randomised, doubleblind, placebo-controlled trial. Lancet Neurol. 2014;13(5):461-71. 